PEB Échanges, Programme pour la construction et l'équipement de l'éducation 2004/08

\title{
Irlande - Des écoles économes en énergie
}

\author{
Martin Heffernan
}

https://dx.doi.org/10.1787/608283442480 


\section{IRLANDE : DES ÉCOLES ÉCONOMES EN ÉNERGIE}

Conformément à son engagement de réduire les émissions de dioxyde de carbone, le ministère irlandais de l'Éducation et de la Science a conçu et construit deux écoles économes en énergie, à Tullamore (comté d'Offaly) et à Raheen (comté de Laois). Comme la consommation d'énergie dans les bâtiments est à l'origine d'environ $55 \%$ du $\mathrm{CO}_{2}$ rejeté dans l'atmosphère qui contribue fortement au réchauffement planétaire, le ministère a étudié les techniques de construction et les systèmes les plus récents pouvant diminuer la consommation énergétique. L'objectif est que les leçons tirées de la construction et de la surveillance de ces bâtiments permettent de réduire l'utilisation d'énergie dans les futurs établissements scolaires. L'entreprise nationale de fourniture d'énergie a financé en partie les systèmes d'économie d'énergie et de surveillance des bâtiments. Les plans d'une troisième école économe en énergie sont en cours de réalisation.

Les avantages liés aux écoles économes en énergie ne se limitent pas à une réduction des émissions de $\mathrm{CO}_{2}$. Tout au long de leur vie, les bâtiments scolaires de Gaescoil et Raheen auront une incidence sur l'environnement bien inférieure à celle des constructions traditionnelles et leurs occupants auront la satisfaction de savoir que leur bâtiment respecte l'environnement. Les immeubles économes en énergie améliorent également le confort en augmentant l'éclairage naturel et les possibilités de programmer la ventilation naturelle. En outre, ces écoles constituent le lieu idéal pour sensibiliser les élèves aux techniques de construction respectueuses de l'environnement.

Ces écoles répondent à un objectif : offrir des équipements éducatifs de qualité, adaptés aux besoins de leurs utilisateurs. Ces projets ne se réduisent pas à une architecture économe en énergie ; il s'agit également de faire remonter au ministère de l'Éducation et de la Science les données sur les écoles et le fonctionnement de leurs systèmes et $d^{\prime}$ utiliser les bâtiments comme des réserves actives d'information sur la conservation de l'énergie et la durabilité.

Des logiciels sophistiqués de simulation de l'énergie et de la lumière naturelle ont permis aux concepteurs de mieux comprendre comment les bâtiments pouvaient réagir à leur environnement et comment leurs réactions pouvaient être utilisées pour améliorer l'environnement scolaire tout en minimisant la consommation d'énergie.

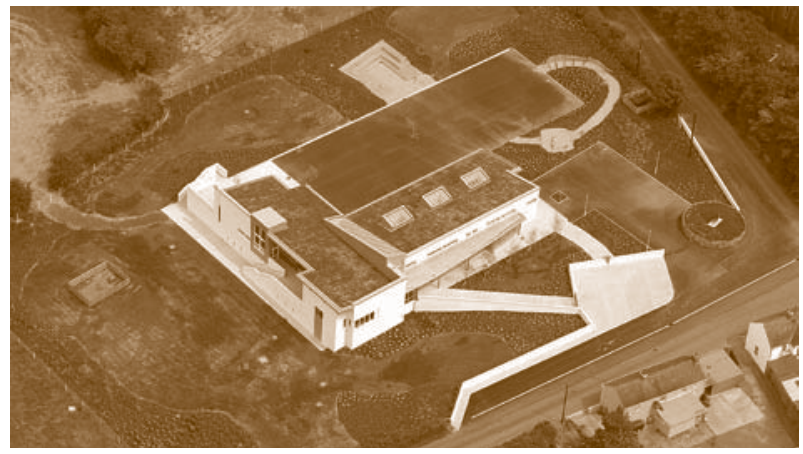

Gaelscoil An Eiscir Riada, Tullamore, comté d'Offaly

\section{Gaelscoil An Eiscir Riada, Tullamore, comté d'Offaly}

Cette école primaire de huit classes a été conçue de manière à consommer $20 \%$ de moins d'énergie qu'un établissement comparable construit selon les normes de bonnes pratiques actuelles, de sorte que le fonctionnement de ses services ne produise pas de dioxyde de carbone et que sa construction reflète un souci de durabilité. Les bâtiments ont été terminés et mis en service en 2003.

L'établissement Gaelscoil est équipé d'un système de surveillance approfondie qui fournit des informations capitales pour comprendre la consommation d'énergie et d'eau dans les bâtiments scolaires. Dans les toutes prochaines années, le bâtiment et ses systèmes feront l'objet d'une surveillance minutieuse. Un système évolué de surveillance de la gestion du bâtiment a été installé afin de recueillir des informations sur le comportement du bâtiment et d'aider ses occupants à affiner les programmations pour minimiser les déperditions d'énergie.

Afin de réduire la consommation, les concepteurs ont veillé à réduire les infiltrations, à choisir des matériaux appropriés, à utiliser la ventilation et la lumière naturelles et à installer un système de récupération des eaux pluviales et un système de chauffage évolué. Un écran tactile renseigne les occupants sur l'énergie consommée par l'école.

À l'origine de déperditions thermiques, les infiltrations d'air sont généralement une source importante de gaspillage d'énergie, en particulier la nuit, quand les bâtiments sont inoccupés. La chaleur s'évacue lentement vers l'extérieur et il faut dépenser plus d'énergie le lendemain pour remettre le bâtiment à température. L'étanchéité à l'air a été vérifiée en injectant de l'air sous pression et en mesurant l'infiltration. Des essais d'étanchéité aux fumées ont été réalisés afin de repérer les voies d'infiltration. Pour diminuer les pertes d'énergie de la structure, les niveaux $d^{\prime}$ 'isolation thermique ont été doublés par rapport aux niveaux prescrits par le code du bâtiment. 


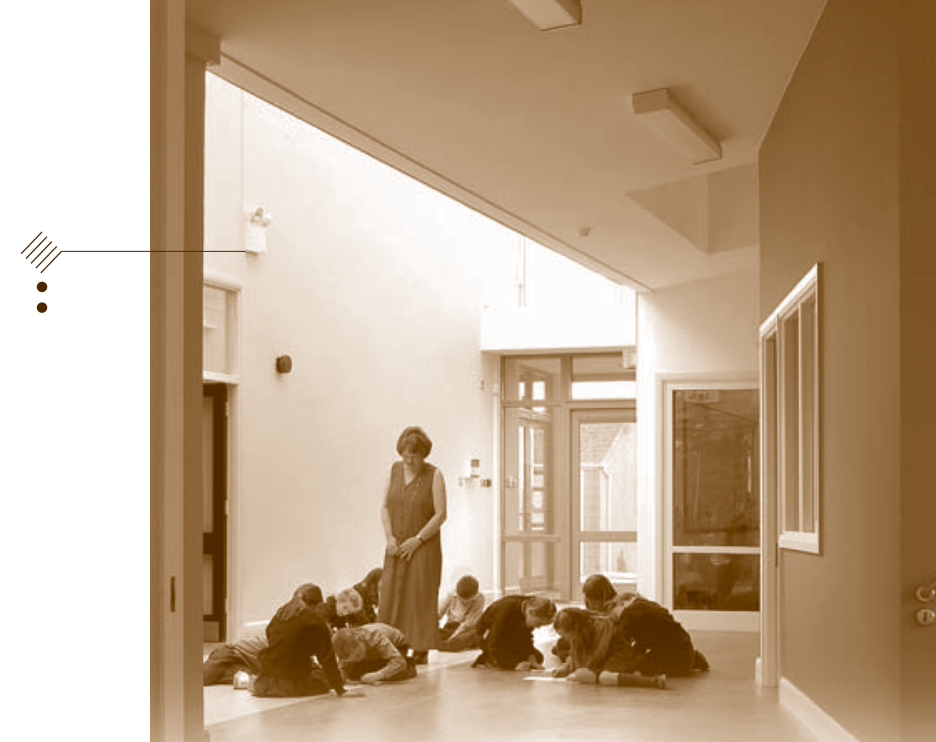

II a été tenu compte de l'énergie consommée pendant les travaux de construction et des matériaux à mettre en œuvre pour respecter l'environnement. Le choix s'est porté sur une structure légère, avec charpente en bois d'œuvre, afin de réduire au minimum l'impact sur l'environnement.

Une attention particulière a été portée à l'utilisation d'une ventilation naturelle adéquate. Les ingénieurs ont réalisé des simulations précises pour optimiser la répartition de l'air dans les classes.

Les plans ont été tracés de sorte que la plupart des fenêtres soient orientées à l'est, pour bénéficier du chauffage gratuit fourni par le rayonnement solaire. Utiliser la lumière du jour dans toutes les classes permet également de ne pas avoir à éclairer avec les lampes, et donc à consommer de l'énergie, durant une très grande partie de l'année. Pendant la journée et au moins 80 \% de l'année, l'éclairage artificiel ne devrait pas être nécessaire dans les classes. La conception des fenêtres a été soigneusement calculée pour garantir des niveaux d'éclairage naturel corrects. De plus, des commandes d'éclairage évoluées ont été installées pour éviter que les lampes ne restent inutilement allumées.

Les eaux de pluie récupérées sur le toit du bâtiment alimentent les chasses d'eau.

Le système de chauffage du bâtiment fonctionne avec une pompe à chaleur géothermique, qui minimise les émissions de $\mathrm{CO}_{2}$. L'eau arrive à la pompe à chaleur après avoir cheminé dans des conduites enterrées à l'extérieur du bâtiment. La pompe restitue au bâtiment la chaleur que le sol, véritable capteur solaire géant, a emmagasinée. Un système de chauffage sous plancher est utilisé pour abaisser la température au départ de la pompe à chaleur. Il stocke la chaleur, permettant de faire fonctionner la pompe de nuit, quand les tarifs sont plus avantageux. Les performances du système de chauffage font l'objet d'une surveillance rigoureuse.

L'électricité nécessaire au fonctionnement de la pompe à chaleur et au reste du bâtiment de Gaelscoil est produite par un groupe éolien. II n'y a donc aucune production de dioxyde de carbone.

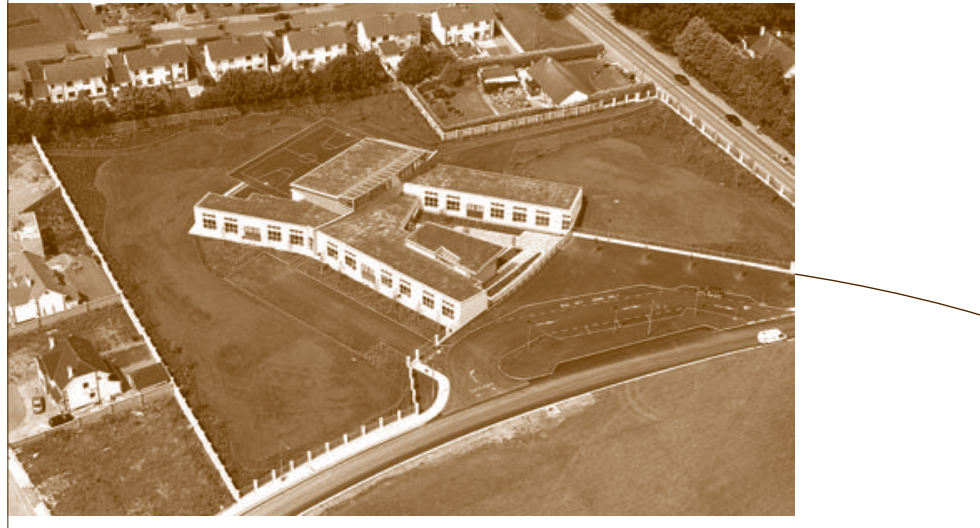

Raheen National School, Raheen, comté de Laois

Le projet Gaelscoil comprend un écran tactile près de l'entrée de l'établissement. Relié au système de gestion de l'énergie, il fournit aux élèves et aux visiteurs des informations sur la consommation d'énergie et l'environnement du bâtiment. Un petit personnage apparaît à l'écran pour encourager les enfants à se renseigner sur la construction du bâtiment et l'utilisation quotidienne de l'énergie. Cet affichage est également relié au système informatique de l'école et peut être consulté depuis n'importe quel ordinateur de l'établissement pour obtenir des informations sur la gestion de l'énergie dans le bâtiment, dans le cadre de projets scolaires.

\section{Raheen National School, Raheen, comté de Laois}

La Raheen National School est une nouvelle école de trois classes conçue en conformité avec le principe de durabilité. Le bâtiment favorise une faible consommation d'énergie et de bons niveaux d'éclairage naturel en tenant compte de l'isolation, des infiltrations d'air, des eaux de pluie, du chauffage, des méthodes d'éclairage et de la couverture. Cette école fonctionne depuis 2003.

Une modélisation thermique dynamique sophistiquée a été réalisée au moyen d'un programme mis au point par le Lawrence Berkeley National Laboratory (États-Unis). Plus de 150 permutations et combinaisons de configuration du bâtiment et des systèmes ont été simulées et les coûts d'équipement et de fonctionnement des différents scénarios ont été comparés. La plupart des recommandations qui en ont résulté avaient trait à l'architecture, l'objectif étant de réduire les besoins en énergie par des apports solaires passifs, la lumière naturelle, etc. L'orientation du bâtiment a été étudiée, ainsi que de nombreuses constructions avec différents types d'isolation.

La structure est en bois d'œuvre, ce qui permet une plus grande épaisseur d'isolation que dans les constructions classiques avec murs creux. Les murs sont isolés par projection de cellulose (journaux recyclés) après étanchéification du bâtiment. Au terme d'une étude sur les avantages 


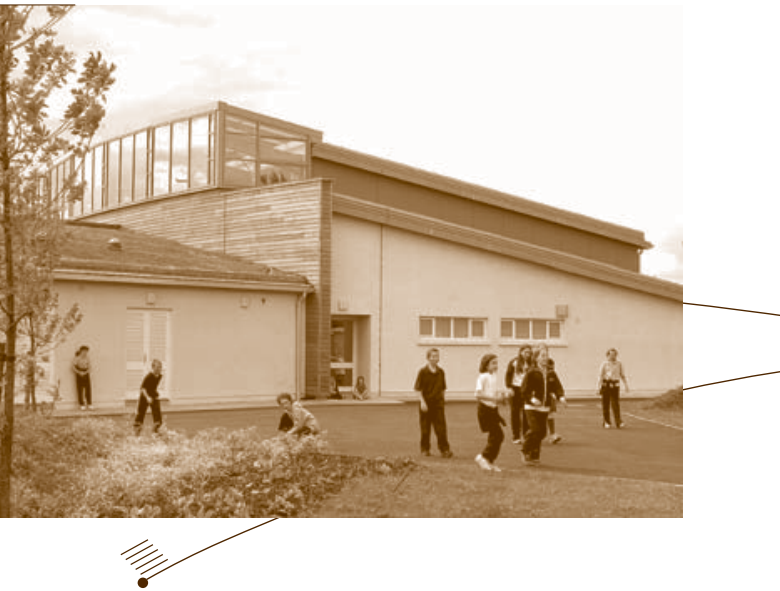

respectifs des structures lourdes et des structures légères, le choix s'est porté sur une structure légère. Cette solution est en effet plus intéressante, dans la mesure où l'école est moins utilisée, en termes d'heures quotidiennes et de jours de l'année, que d'autres bâtiments institutionnels. La structure légère est également préférable car elle permet au bâtiment de réagir plus vite à son système de chauffage, c'est-à-dire de se réchauffer et de profiter de l'accumulation solaire plus vite. A contrario, avec des murs contenant une lourde plaque intérieure, il faudrait une journée entière pour stocker la chaleur solaire, qui, en outre, ne serait restituée qu'après le départ des élèves en fin de journée.

Les ingénieurs en mécanique et en électricité ont observé que, phénomène typique, une grande partie de l'énergie de chauffage d'un bâtiment sert à chauffer l'air qui s'infiltre. Dans le cadre du cahier des charges architecturales, un coefficient d'infiltration d'air et un essai de vérification de l'étanchéité à l'air avaient été préconisés. L'indice d'infiltration prescrit était de $5 \mathrm{~m}^{3} / \mathrm{h} / \mathrm{m}^{2}$ sous une pression de 50 Pa.

Des essais de pression ${ }^{1}$ ont été réalisés avec du matériel spécialisé. Le but de ces essais est de détecter les voies d'infiltration et de les neutraliser. Le premier essai a mis en évidence des taux supérieurs à la valeur prescrite. Pendant l'essai, une fumée artificielle a été injectée dans le bâtiment et les endroits par lesquels elle s'échappait ont mis en évidence les principales voies d'infiltration. Un essai ultérieur a donné des résultats conformes au cahier des charges.

Raheen utilise les eaux pluviales pour les sanitaires. L'eau est récupérée sur le toit et filtrée avant d'être stockée dans une citerne conçue à cet effet. Par temps sec, la citerne se vide jusqu'à un certain point au-delà duquel un capteur de niveau déclenche le raccordement au réseau d'adduction d'eau, afin de maintenir un niveau minimum. La quantité d'eau totale consommée par les toilettes et la quantité prélevée sur le réseau sont enregistrées toutes les heures par un système de gestion du bâtiment en liaison avec les ingénieurs du ministère de l'Éducation et de la Science à Tullamore (comté d'Offaly). Les résultats indiquent si le

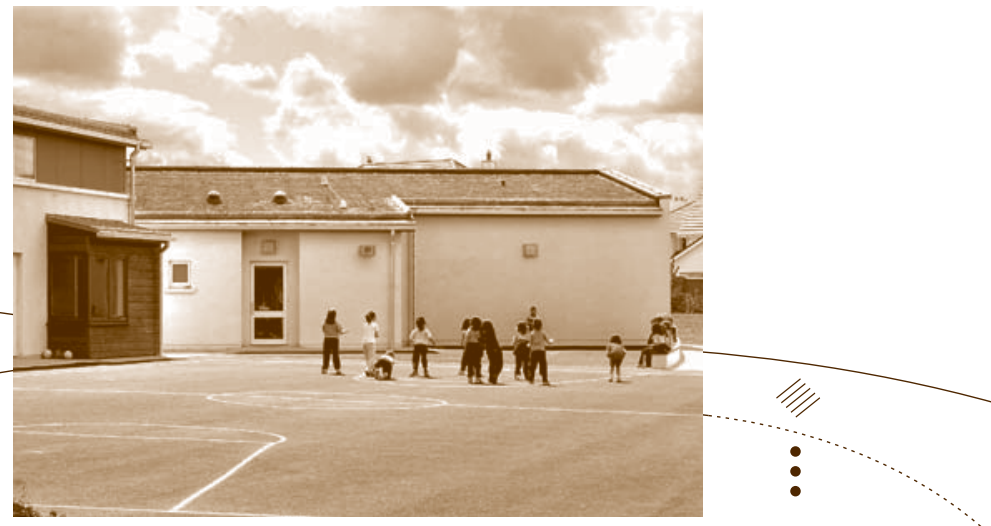

système de récupération est efficace et donnent une idée de la quantité d'eau consommée pour les sanitaires. Les arguments qui justifient de récupérer l'eau de pluie pour remplir les chasses d'eau sont solides : ce système de récupération permet de moins solliciter le réseau d'adduction et le système d'évacuation des eaux pluviales. Une simulation très précise a été effectuée avec différentes tailles de citerne et une modélisation spécifique au projet a été réalisée : elle sert à estimer la demande horaire et la quantité d'eau de pluie disponible tout au long de la journée.

Avant de choisir un système de chauffage pour le bâtiment et l'eau domestique, différentes comparaisons de systèmes mécaniques ou électriques ont été effectuées :

- chauffage au fuel par convecteurs vs pompe à chaleur géothermique couplée à un système de chauffage sous plancher ;

- centrale de chauffage modulaire avec une à quatre chaudières ;

- chauffage de l'eau par pompe à chaleur vs chauffage de I'eau domestique par un système de chauffage central au fuel vs chauffage de l'eau domestique par un système électrique décentralisé ;

- commandes de régulation en fonction de la température.

Les comparaisons ont montré que, en l'absence de gaz naturel, la solution qui produirait le moins d'émissions de $\mathrm{CO}_{2}$ était le système comprenant des chaudières au mazout classiques et des radiateurs. L'une des chaudières sert à chauffer à la fois le bâtiment et l'eau domestique.

L'éclairage est assuré par des lampes à haute fluorescence ; toutefois, pour réduire la consommation de lumière arti-

1. Les avis sont partagés en ce qui concerne les essais de pression sur des bâtiments scolaires. Pour certains, une école où l'air s'infiltre gaspille de l'énergie ; pour d'autres, il faut une ventilation de fond permanente pour réduire les risques de condensation. La société d'ingénierie Overy and Associates qui a travaillé à Raheen considère que c'est un faux problème car les ouvertures assurant une ventilation de fond permanente existent en quantité déterminée. 
ficielle, une commande à cellule photoélectrique a été installée : elle diminue automatiquement l'intensité des lampes ou les éteint quand la lumière du jour est suffisante. Toutes les lampes linéaires sont équipées de ballasts électroniques. Celles qui se trouvent près des fenêtres ont des ballasts à gradation, l'intensité étant commandée par une cellule photoélectrique au plafond. Les lampes normales et les lampes à gradation sont commandées par des interrupteurs distincts. Après avoir réfléchi à plusieurs formules avec variateur automatique, les concepteurs ont opté pour une extinction manuelle des lampes quand la lumière du jour suffit.

Les espaces de circulation, non couverts, sont principalement éclairés par la lumière du jour. Dans le couloir et le hall, des cellules photoélectriques allument automatiquement les lampes quand l'éclairement lumineux naturel descend au-dessous de 150 lux. Les couloirs sont si bien éclairés par les lucarnes que les lampes ne sont allumées que durant les journées les plus sombres. Les interrupteurs sont munis d'indicateurs au néon rappelant qu'il faut couper les circuits la nuit.

Dans les locaux de stockage, les lampes sont équipées de détecteurs de présence à infrarouge. II n'y a aucun interrupteur et il est donc impossible d'oublier d'éteindre en sortant.

À l'extérieur, l'éclairage est dispensé par des lampes compactes à fluorescence et des lampes à sodium à haute pression. Les lampes éclairant les entrées et les sorties sont déclenchées par une cellule photoélectrique et reliées à une minuterie. Ainsi, elles ne s'éclairent qu'à la tombée du jour mais ne restent pas allumées toute la nuit. L'éclairage de sécurité est assuré par des projecteurs avec détecteur d'intrusion à infrarouge.

Les matériaux de couverture ont été sélectionnés de façon à concilier propriétés écologiques, rendement énergétique et commodité. La toiture, du même type que celle de Gaelscoil, est une structure légère en bois vert qui augmente l'isolation de la construction. En outre, le toit a un faible contenu énergétique, transforme en permanence le $\mathrm{CO}_{2}$ par photosynthèse et sert de support pédagogique car il abrite des micro-écosystèmes. L'orpin qui le recouvre contribue à atténuer les effets de la pluie et à absorber les bruits extérieurs.

\section{Article de Jeff Colley, Construct Ireland}

Pour plus d'informations sur la conception et la construction durables, voir le site www.constructireland.ie ou contacter :

\section{Martin Heffernan}

Planning and Building Unit

Department of Education and Science

Tullamore, Co. Offaly, Irlande

Télécopie : 35350651119

martin_heffernan@education.gov.ie

\section{AUSTRALIE : PREMIER PROJET DE PARTENARIAT PUBLIC/PRIVÉ POUR LA CRÉATION D'ÉCOLES}

En Australie, la conception et la construction de neuf écoles commence, sous l'égide d'un partenariat public/ privé (PPP). C'est la première fois qu'un projet de ce type, portant sur l'acquisition d'une infrastructure sociale, est conduit dans ce pays.

Au Royaume-Uni et dans tous les pays de l'Union européenne, il est fréquent de créer des PPP pour financer des infrastructures sociales qui génèrent des revenus pour leur exploitant privé (routes à péage, projets ferroviaires ou ponts, etc.). Cependant, des projets de plus en plus nombreux concernent la construction d'hôpitaux, de prisons et d'écoles, c'est-à-dire d'infrastructures qui ne rapportent (pratiquement) aucun revenu à leur promoteur, celui-ci n'étant rémunéré que par l'État.

Le projet australien est dirigé par le ministère de l'Éducation et de la Formation de l'état de Nouvelle-Galles du

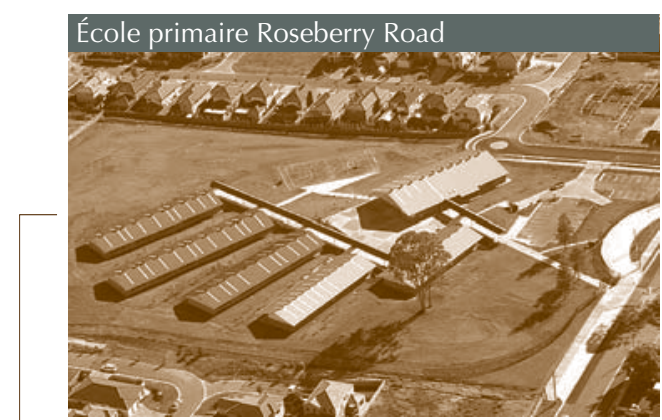

